\title{
ALEITAMENTO INTENSIFICADO PARA BEZERROS DA RAÇA HOLANDESA: DESEMPENHO, CONSUMO, CONVERSÃO ALIMENTAR E ESCORE DE CONSISTÊNCIA FECAL
}

\author{
Ivan Pedro de Oliveira Gomes ${ }^{1}$, André Thaler Neto ${ }^{1}$, Helder de Arruda Córdova ${ }^{1}$, \\ Roberto Parizotto Filho ${ }^{1}$, Marciel França ${ }^{1}$, Eduardo Eriberto Simon ${ }^{1}$ \\ 1 UDESC \\ Correspondência Ivan Pedro de Oliveira Gomes: ivan.gomes@udesc.br
}

RESUMO: O presente trabalho teve por objetivo avaliar o consumo de alimentos, conversão alimentar, desempenho, escore de consistência fecal e dias com diarréia de bezerros submetidos a estratégias de aleitamento intensificado e convencional. O experimento foi realizado no setor de bovinocultura de leite do CAV/UDESC, em Lages - SC. Foram utilizados 24 bezerros da raça Holandesa em um delineamento experimental em blocos ao acaso. Após o período de colostro foram distribuídos em dois tratamentos: I - Convencional, com $4 \mathrm{Kg}$ de leite por dia; II - Intensificado, na base de $14 \%$ do peso vivo ajustado semanalmente ao peso dos mesmos. Os bezerros foram desmamados com 8 semanas de idade, sendo que na semana anterior ao desmame o fornecimento de leite foi reduzido à metade. Os animais tiveram acesso a um concentrado inicial farelado com $18 \%$ de PB e água à vontade até 56 dias de idade. Do desmame até 16 semanas de idade receberam uma mistura do concentrado inicial, feno de alfafa e água. Os dados foram submetidos à análise de variância. Os bezerros alimentados com maiores quantidades de leite (7,38 litros por dia) apresentaram um menor consumo de concentrado durante o período de aleitamento, maior ganho de peso diário, maior peso vivo e maior altura em relação aos animais aleitados com quantidades convencionais de leite ( $4 \mathrm{~L}$ por dia). Entretanto após este período (9-16 semanas) não houve diferença entre os tratamentos. Apesar de ser observado um ligeiro aumento no escore de consistência fecal, isto não implicou em um aumento na incidência de diarreia.

Palavras-chave: altura na cernelha; ganho em altura, ganho de peso; ingestão de concentrado; peso vivo

\section{INTENSIFIED FEEDING FOR HOLSTEIN CALVES: PERFORMANCE, INTAKE, FEED CONVERSION AND FECAL CONSISTENCY SCORE}

\begin{abstract}
This study aimed to evaluate the feed intake, feed conversion, performance, fecal consistency score and days with diarrhea of calves subjected to conventional and intensified strategies of milk feeding management. The experiment was conducted at the Dairy Cattle Sector of CAV/UDESC, Lages - SC, Brazil. Twenty four Holstein calves was used in a experimental trial in randomized blocks. After receive the colostrum they were assigned to two treatments: conventional, with $4 \mathrm{~kg}$ of milk per day and intensive, on the basis of $14 \%$ of body weight adjusted weekly to their weight. Calves were weaned at 8 weeks of age and in the last week before the wean the milk supply was cut to half. The animals had access to a calf starter with $18 \% \mathrm{CP}$ and water ad libitum until 56 days of age. From the wean to 16 weeks of age they received calf starter, alfalfa hay and water. The data were submitted to ANOVA. Calves fed with more milk (7,38 liters a day) showed less feed concentrate intake during the milk-feeding period, higher daily weight gain, liveweight, and height at withers to conventional group. However, after this period (9-16 weeks) there was no difference between treatments. Instead had observed a slight enhanced on fecal consistency score, the days with diarrhea were not affect.
\end{abstract}

Key Words: height at withers; height gain, weight gain; intake of concentrate; liveweight 
Aleitamento intensificado para bezerros da raça holandesa:

desempenho, consumo, conversão alimentar e escore de consistência fecal

\section{INTRODUÇÃO}

Um sistema eficiente de criação de bezerras e novilhas, incluindo o manejo alimentar no período de aleitamento é fundamental, pois poderá determinar a sustentabilidade e a rentabilidade futura da atividade leiteira. No sistema convencional, os bezerros são separados das mães após o nascimento e alimentados com uma quantidade controlada de colostro e, em seguida com 4 litros de leite ou sucedâneo por dia (sistema convencional), equivalente a $8-10 \%$ do peso vivo até 0 desaleitamento (KHAN et al., 2007a; SILPER, 2012). Porém, as bezerras tem potencial para um crescimento mais rápido do que ocorre normalmente na maioria das explorações leiteiras sem acúmulo excessivo de gordura corporal e comprometimento da produção de leite e de sólidos (BROWN et al., 2005). Um bezerro ao pé da vaca normalmente mama 6 a 10 vezes ao dia e consome o equivalente a 16 a $24 \%$ do peso vivo em leite. Isto representa 2 a 3 vezes o que normalmente se fornece num sistema convencional de criação de bezerros leiteiros (DRACKLEY e HOFFMAN, 2002). Portanto o ideal seria que a quantidade de leite ou sucedâneo fosse ajustada conforme o peso vivo do animal.

A quantidade e o método de alimentação dos bezerros com leite têm enormes efeitos sobre 0 seu desempenho, comportamento, saúde e bem-estar (KHAN et al., 2007b). Os autores acima citados observaram maior consumo de leite e alimentos sólidos, ganho de peso vivo e a eficiência alimentar (EA) em bezerros criados em um sistema intensificado. Melhorias no regime nutricional do bezerro pode diminuir a mortalidade e susceptibilidade às doenças e aumentar 0 ganho de peso diário (BALDWIN et al., 2004). A alimentação restrita com leite geralmente deprime o crescimento do bezerros (JASPER e WEARY, 2002).

$O$ crescimento intensificado antes da puberdade é uma forma de diminuir a idade ao primeiro parto sem interferir no desenvolvimento da glândula mamária (BROWN et al., 2005). Portanto para atingir idade mais baixa na puberdade $e$ no primeiro parto em novilhas leiteiras, 0 período de pré-desmama oferece um potencial para aumentar as taxas de crescimento através do aumento da ingestão de energia e proteína sem causar engorda excessiva. A nutrição e o manejo no pré desmame é um dos principais fatores que influênciam a expressão do potencial genético do animal quanto a produção de leite (SOBERON et al., 2012).

Recentemente vários trabalhos têm avaliado a fornecimento de leite na proporção de 15 a $20 \%$ do peso vivo (sistema intensificado) a e sua influência no consumo de alimentos, ganho de peso diário (GPD), estatura, incidência de doenças (diarréia), desenvolvimento da glândula mamária e produção de leite nas primeiras lactações. Estes estudos tem revelado um maior consumo de leite e ganho de peso vivo dos animais criados neste sistema em comparação com 0 sistema convencional de fornecimento de leite. Jasper e Weary (2002) relataram $89 \%$ a mais de consumo de leite e $63 \%$ a mais de GPD ao desmame de bezerros alimentados ad libitum que aqueles convencionalmente. Estes autores observaram uma redução no consumo de concentrado inicial no sistema intensificado. Resultados semelhantes foram relatados por Khan et al. (2007a, 2007b). Segundo Rincker et al. (2011) bezerras consumindo altos níveis de energia e proteína durante o período pré desmame tem peso vivo, altura na cernelha e largura da garupa ao desmame maior que as bezerras que 
consomem dieta convencional. Segundo esses mesmos autores as bezerras que recebem alimentação intensificada apresentam escore fecal alto, porém não foi observada diarréia. Os autores acima citados relataram que as novilhas alimentadas com dietas intensificadas alcançaram a puberdade mais novas e peso mais leve. Em relação ao custo de produção não houve diferença significativa entre os tratamentos. Resultados semelhantes foram relatados por Raeth-Knight et al. (2009) e Brown et al. (2005), onde economicamente, os dois programas (convencional e intensificado) não foram significativamente diferentes, indicando que as fazendas podem considerar outros fatores específicos de manejo para a adoção do sistema intensificado.

Raeth-Knight et al. (2009) ao avaliarem a produção de leite de novilhas que foram alimentadas com 0 uso intensificado de sucedâneos não encontraram diferença em relação a produção de leite na primeira lactação para novilhas criadas com o sistema convencional. A única diferença encontrada foi em relação à idade ao parto, sendo que as novilhas que foram amamentadas através do sistema intensificado pariram 27,5 dias antes que as alimentadas no sistema tradicional. Morrisson et al. (2009) também não encontraram diferença na produção de leite na primeira lactação ao comparar a criação de bezerras nos dois sistemas.

O presente trabalho teve por objetivo avaliar os efeitos da alimentação intensificada sobre 0 crescimento de bezerros da primeira até a $16^{\underline{a}}$ semana de vida.

\section{MATERIAL E MÉTODOS}

O trabalho foi desenvolvido no projeto de bovinocultura de leite do Centro de Ciências Agroveterinárias/UDESC, de Lages, SC.
Foram utilizados 24 bezerros (12 machos e 12 fêmeas) da raça Holandesa. Os animais receberam colostro até o quinto de dia de vida. Após foram distribuídos em dois tratamentos: I - Convencional aleitamento com leite natural na base de $4 \mathrm{Kg}$ por dia (10\% peso vivo ao nascer) fornecidos duas vezes ao dia; II intensificado - alimentados com leite natural na base de $14 \%$ do peso vivo ajustado semanalmente conforme o peso da semana anterior. Os bezerros foram desmamados com 8 semanas de idade. Na semana anterior ao desmame (sétima) foi fornecida a metade do leite calculada para o sistema intensificado. Os animais tiveram acesso a um concentrado inicial farelado com $18 \%$ PB (Tabela 1) e água a vontade até 56 dias de idade. Do desmame até as 16 semanas de idade receberam 0 concentrado inicial à vontade mais feno de alfafa e água.

\begin{tabular}{lcc}
\hline \multicolumn{2}{c}{ Tabela 1 - Composição dos alimentos fornecidos aos bezerros (\% da MS). } \\
\hline & Ração Inicial & Feno de alfafa \\
\hline Proteína Bruta & 19,6 & 16,7 \\
Extrato Etéreo & 4,2 & 2,8 \\
FDN & 29,6 & 51,2 \\
FDA & 17,6 & 37,9 \\
Matéria Mineral & 11,9 & 10,2 \\
\hline
\end{tabular}

Os bezerros foram alojados em bezerreiro com baias individuais de $1 \mathrm{x}$ $1,5 \mathrm{~m}$ com piso ripado de madeira. $\mathrm{O}$ leite foi fornecido em mamadeira com capacidade para 2 litros, logo após a ordenha (2 vezes ao dia). Foram registrados peso vivo $(\mathrm{kg})$ e altura na cernelha $(\mathrm{cm})$ semanalmente, antes do fornecimento do leite. $O$ concentrado e o feno foram fornecidos diariamente $e$ as sobras recolhidas a cada dois dias e pesadas. Foram avaliados o consumo de leite, concentrado, volumoso, conversão alimentar (kg MS ingerida / $\mathrm{kg}$ de ganho de peso), escore da consistência fecal conforme metodologia 
Aleitamento intensificado para bezerros da raça holandesa: desempenho, consumo, conversão alimentar e escore de consistência fecal

descrita por Larson et al. (1977) e dias com diarreia.

O delineamento experimental utilizado foi 0 de blocos ao acaso, considerando-se como bloco o sexo dos animais. Os dados obtidos foram submetidos à análise de variância utilizando-se o procedimento GLM do pacote computacional SAS ${ }^{\circledR}$.

\section{RESULTADOS E DISCUSSÃO}

O consumo total de leite durante o período experimental foi de 196 litros para os bezerros com aleitamento convencional, com média de 4 litros/dia, e de 361,62 litros para o sistema intensificado, equivalente a 7,38 litros/dia, com incremento de $84,5 \%$ no consumo de leite em relação ao aleitamento convencional (Tabela 2). $\mathrm{O}$ aumento do consumo de leite foi similar aos valores observados no trabalho de Jasper e Weary (2002), no qual os bezerros recebendo leite ad libitum consumiram em média 8,8 litros ao dia, comparado com 4,7 litros/dia consumidos pelos bezerros alimentados no sistema convencional e no trabalho de Khan et al. (2007b), no qual o consumo no período de 1 a 49 dias foi de 7,84 e 4,74 litros, respectivamente.

O consumo de concentrado da $1^{\text {a }}$ à 8 a semana apresentou-se menor $(\mathrm{P}<0,01)$ no aleitamento intensificado, com consumo médio de $0,361 \mathrm{~kg}$ contra 0,697 kg do grupo controle (Tabela 2). Apesar deste consumo não diferir no segundo período (9 a à 16 ${ }^{\underline{a}}$ semana), no período experimental total houve uma tendência de maior consumo por parte do grupo controle $(P=0,078)$. RaethKnight et al. (2009) observaram que bezerros recebendo uma quantidade maior de dieta líquida diminuíram a ingestão de concentrado inicial do nascimento até 49 dias de idade. Jasper e Weary (2002) relatam o consumo de aproximadamente 0 dobro de concentrado pelo grupo recebendo $10 \%$ do peso vivo de leite comparado a bezerros em aleitamento à vontade. Resultado semelhante foi observado por Khan et al. (2007b) no período de 1 a 28 dias de aleitamento. Mesmo com redução do consumo de concentrado no período de aleitamento devido a maior quantidade de leite fornecida, o consumo de alimentos sólidos após o desaleitamento foi semelhante (Tabela 2), podendo ser até mesmo superior ao consumo de bezerros aleitados de forma convencional (JASPER e WEARY, 2002; KHAN et al., 2007a).

\begin{tabular}{|c|c|c|c|c|c|}
\hline \multirow{2}{*}{ Variáveis } & \multicolumn{2}{|c|}{ Tratamentos } & \multirow{2}{*}{$\begin{array}{c}\text { Valor de } \\
\mathbf{P}\end{array}$} & \multirow{2}{*}{$\begin{array}{l}\text { CV } \\
(\%)\end{array}$} & \multirow{2}{*}{ EPM } \\
\hline & Controle & Intensificado & & & \\
\hline \multicolumn{6}{|l|}{ Leite total, Iitros } \\
\hline 1-8 semanas & 196,00 & 361,62 & - & - & - \\
\hline \multicolumn{6}{|l|}{ Leite, média $/ \mathrm{kg} / \mathrm{dia}$} \\
\hline 1-8 semanas & 4,0 & 7,38 & - & - & - \\
\hline \multicolumn{6}{|l|}{ Concentrado, kg/dia } \\
\hline 1-8 semanas & 0,697 & 0,361 & 0,0042 & 45,84 & 0,07 \\
\hline $9-16$ semanas & 2,586 & 2,420 & 0,3545 & 15,74 & 0,11 \\
\hline $1-16$ semanas & 1,684 & 1,456 & 0,0782 & 17,55 & 0,08 \\
\hline \multicolumn{6}{|l|}{ Feno, kg/dia } \\
\hline $8-16$ & 0,613 & 0,567 & 0,2845 & 16,03 & 0,03 \\
\hline \multicolumn{6}{|l|}{$\mathrm{CA}^{1}, \mathrm{~kg} \mathrm{MS} / \mathrm{kg}$ ganho } \\
\hline $1-8$ semanas & 1,832 & 1,693 & 0,2011 & 14,05 & 0,071 \\
\hline 9-16 semanas & 3,235 & 3,085 & 0,3170 & 10,48 & 0,095 \\
\hline $1-16$ semanas & 2,551 & 2,439 & 0,2939 & 9,46 & 0,068 \\
\hline
\end{tabular}

Não houve diferença no consumo de feno de alfafa (Tabela 2), o qual foi fornecido apenas no período pósdesaleitamento. Jasper e Weary (2002) não observaram diferenças em consumo de alimentos sólidos (concentrado e feno) no período pósdesaleitamento em bezerros de 43 a 63 dias de idade, apesar do menor consumo durante o aleitamento. Khan et al. (2007a) observaram maior consumo de concentrado e feno no período pósdesaleitamento no grupo de bezerras que recebeu maior quantidade de leite.

A conversão alimentar (CA) não diferiu nos três períodos avaliados (Tabela 2). Raeth-Knight et al. (2009) e Rincker et al. (2011) observaram menor 
CA para bezerras alimentadas com menor quantidade de leite.

$O$ peso vivo na $8^{\text {a }}$ semana foi maior $(P=0,024)$ no aleitamento intensivo, com peso médio de $77,64 \mathrm{~kg}$ contra $71,69 \mathrm{~kg}$ do grupo controle (Tabela 3). A diferença de peso observada nos animais que foram aleitados intensivamente $(+5,95)$ foi devido ao maior ganho de peso diário (GPD) durante o período de 1 a 8 semanas $(0,734$ vs. $0,613 \mathrm{~kg})$. Isto deixa claro que existe oportunidade para alto ganho de peso nas primeiras semanas de vida. Não houve diferença no peso na $16^{\underline{a}}$ semana $(P=0,136)$. $O$ maior peso observado na oitava semana atribui-se a maior ingestão de nutrientes devido ao maior volume de leite consumido $(7,38 \mathrm{~kg})$ pelos animais do sistema intensificado em relação ao grupo controle. Raeth-Knight et al. (2009) reportaram que bezerras submetidas ao sistema convencional apresentaram menor peso vivo durante o período de aleitamento (42 dias) quando comparado com bezerras criadas no sistema intensificado. Estes autores observaram que bezerras recebendo altas quantidades de leite em pó e concentrado inicial apresentaram maior peso e altura no pós-desmame que bezerras alimentadas com quantidade convencional de sucedâneo. Brown et al. (2005) também relataram maior peso vivo e altura de bezerras alimentadas no sistema intensificado quando comparado com sistema convencional.

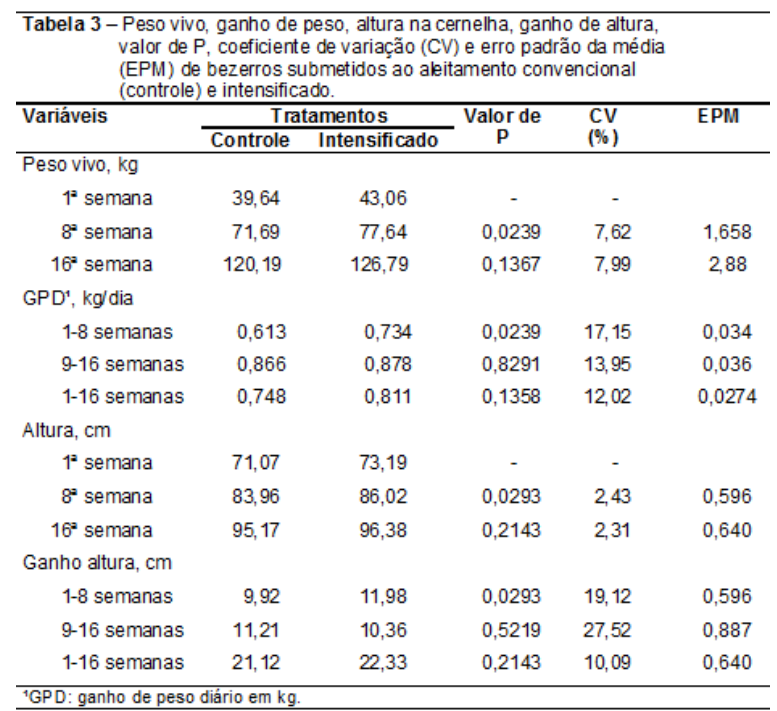

O GPD durante o período de aleitamento também foi maior $(P=0,0239)$ no tratamento intensificado (Tabela 3). Não houve diferença após o desaleitamento e em todo o período experimental em conjunto. Este maior GPD foi consequência da maior ingestão de leite nas primeiras oito semanas, apesar do menor consumo de concentrado observado durante este período. Silper (2012) observou que após 0 desaleitamento não houve diferença quanto ao GPD avaliado a cada semana entre os grupos convencional e intensificado. Jasper e Weary (2002) também não encontraram diferença estatística no ganho de peso pós-desaleitamento entre bezerros que receberam leite à vontade ou a $10 \%$ do PV. Coles et al. (2006) e Khan et al. (2007a) relataram que o ganho de peso foi maior para o grupo aleitado intensivamente tanto no período de aleitamento quanto após o desaleitamento. De acordo com vários autores o GPD do grupo intensificado $(0,811 \mathrm{~kg})$ mostrou-se mais adequado para os padrões de crescimento da raça Holandesa.

Observou-se uma maior altura na cernelha e ganho em altura nos bezerros que receberam tratamento intensificado $(P=0,0293)$ durante 0 período de aleitamento (Tabela 3). Após o desaleitamento não ocorreram 
Aleitamento intensificado para bezerros da raça holandesa:

desempenho, consumo, conversão alimentar e escore de consistência fecal

diferenças tanto na altura na cernelha quanto ao ganho em altura $(P>0,05)$. Rincker et al. (2011) reportaram maior altura no período de aleitamento em bezerras recebendo altos níveis de sucedâneo. Raeth-Knight et al. (2009) observaram diferença em altura na garupa entre tratamentos (convencional $x$ intensificado) somente no período de desaleitamento. Brown et al. (2005) relataram maior altura de bezerras alimentadas no sistema intensivo quando comparado com sistema convencional, em ambos períodos.

Com relação à incidência de diarreia os animais do grupo intensificado apresentaram tendência de maior escore de consistência fecal durante 0 período de aleitamento $(P=0,0643)$ em relação ao grupo controle, não havendo diferenças no número de dias com diarréia (Tabela 4). Isto pode ser devido ao fato de terem consumido maior quantidade de leite. Coles et al. (2006) não observaram diferença no escore fecal, porém os dias com medicação para diarreia foi maior no grupo intensificado. Rincker et al. (2011) constataram que as bezerras que recebem alimentação intensificada apresentam escore fecal alto, apesar de não observarem aumento na incidência de diarréia. Brown et al. (2005) e RaethKnight et al. (2009) reportaram maior escore fecal no período de 2 a 8 semanas nos bezerros alimentados de forma intensificada.

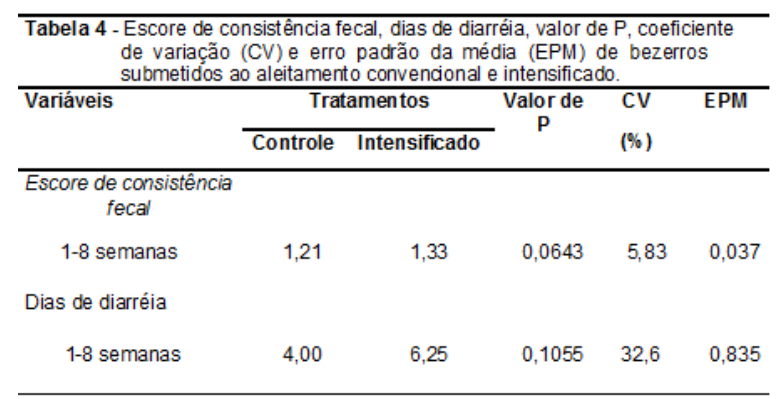

\section{CONCLUSÃO}

A utilização de um sistema de alimentação intensificada no período de aleitamento (leite fornecido na base de $14 \%$ do peso) promoveu um maior desempenho em ganho de peso e altura na cernelha em relação ao aleitamento convencional (10\% do peso). Após o período de aleitamento (9-16 semanas) não foram encontradas diferenças significativas no desempenho dos animais. Portanto, não foram constatados efeitos residuais da alimentação intensiva no período de aleitamento sobre 0 desempenho posterior dos animais;

Os bezerros do grupo intensificado apresentaram um menor consumo de concentrado durante 0 período de aleitamento, porém após este período não houve diferenças entre os tratamentos.

Apesar de ser observado um ligeiro aumento no escore de consistência fecal, isto não implicou em um aumento na incidência de diarreia no grupo que recebeu quantidades maiores de leite.

\section{REFERÊNCIAS}

BALDWIN, R. L. VI; MCLEOD, K. R.; KLOTZ, J. L. et al. Rumen development, intestinal growth and hepatic metabolism in the pre- and postweaning ruminant. Journal of Dairy Science. v.87, n.13, p.55-65, 2004.

BROWN, E. G.; VANDEHAAR, M. J.; K. M. DANIELS, K. M. et al. Effect of Increasing Energy and Protein Intake on Body Growth and Carcass Composition of Heifer Calves. Journal of Dairy Science. v.88, n.2, p.585-594, 2005.

COLES, K. E.; WHITE, R. A.; WHITEHOUSE, N. L. et al. Growth characteristics of calves fed an intensified milk replacer regimen with additional lactoferrin. Journal of Dairy Science. v.89, n.12, p.4835-4845, 2006.

DRACKLEY, J. K., HOFFMAN, P. C. What about intensive calf feeding programs. Hoard's 
Dairyman. v.147, n.6 , p.250-251, march 25, 2002.

JASPER, J.; WEARY, D.M. Effects of ad libtum milk intake on dairy calves. Journal of Dairy

Science. v.85, n.11, p.3054-3058, 2002.

KHAN, M. A.; LEE, H. J.; LEE, W. S. et al. Preand postweaning performance of holstein female calves fed milk through step-down and conventional methods. Journal of Dairy

Science. v.90, n.2, p.876-885, 2007a.

KHAN, M. A.; LEE, H. J.; LEE, W. S. et al. Structural growth, rumen development, and metabolic and immune responses of holstein male calves fed milk through step-down and conventional methods. Journal of Dairy

Science. v.90, n.7, p.876-885, 2007b.

LARSON, L. L., OWEN, F. G., ALBRIGHT, J. L. et al. Guidelines toward more uniformity in measuring and reporting calf experimental data. Journal of Dairy Science, v.60, n.6, p.989-991. 1977.

MORRISON, S. J.; WICKS, H. C. F.; FALLON, R. J. et al. Effects of feeding level and protein content of milk replacer on the performance of dairy herd replacements. The Animal

Consortium. v.3, n.11, p.1570-1579, 2009.

RAETH-KNIGHT, M.; CHESTER-JONES, H.; HAYES, S. et al. Impact of conventional or intensive milk replacer programs on Holstein heifer performance through six months of age and during first lactacion. Journal of Dairy Science. v.92, n.2, p.799-809, 2009.

RINCKER, L. E. D.; VANDEHAAR, M. J.; WOLF, C. A. et al. Effect of intensified feeding of heifer calves on growth, pubertal age, calving age, milk yield, and economics. Journal of Dairy

Science. v.94, n.7, p.3554-3567, 2011.

SAS_INSTITUTE. SAS/STAT User's Guide 8.0. Cary-NC: SAS Institute. 1999.

SILPER, B. F. Efeitos de três estratégias de aleitamento sobre ganho de peso, desenvolvimento ruminal e perfil metabólico e hormonal de bezerros holandeses. 2012 . 96p. Dissertação (Mestrado) - Universidade Federal de Minas Gerais, Belo Horizonte, 2012.

SOBERON, F.; RAFFRENATO, E.; EVERETT, R. W. et al. Preweaning milk replacer intake and effects on long-term productivity of dairy calves. Journal of Dairy Science. v.95, n.2, p.783-793, 2012. 